\section{LETTERS TO THE EDITOR}

If you have a burning desire to respond to a paper published in Thorax, why not make use of our "rapid response" option? Log on to our website (www.thoraxjnl.com), find the paper that interests you, and send your response via email by clicking on the "eletters" option in the box at the top right hand corner.

Providing it isn't libellous or obscene, it will be posted within seven days. You can retrieve it by clicking on "read eletters" on our homepage.

The editors will decide as before whether to also publish it in a future paper issue.

\section{Duplicate publication}

We are writing to express our unease at what we believe is inappropriate censure imposed on our colleague Professor Corris concerning duplicate publications. ${ }^{12}$ Professor Corris was asked to write what was essentially a CME article for Clinical Medicine on a subject that he had recently reviewed in detail for Thorax. I was inevitable that there would be considerable duplication. The same papers and information were being disucssed and there are limitations in the way complex arguments can be expressed. It is universally accepted that a degree of duplication in review articles is completely different from trying to pass off as a new study previously published pee reviewed papers containing original data. It is commonplace for people with authoritative opinions to write similar articles in more than one journal as shown by the similarities between the Harveian oration by Warrell published in the same issue of Clinical Medicine and an earlier manuscript in the Lancet. ${ }^{34}$ We believe such duplication is entirely appropriate, as surely it is our duty as educators to disseminate information to as wide an audience as possible. Fraud in any shape or form in science is to be wholly deplored, but let us not be so zealous in its pursuit that we smear the innocent to the detriment of us all.

At risk of another duplicate publication, we have also sent this letter to the editor of Clinical Medicine.

I D Pavord, M D L Morgan, A J Wardlaw Institute for Lung Health, Department of Respiratory Medicine and Thoracic Surgery, Glenfield Hospital, Groby Road, Leicester LE3 9QP UK;trina.raftery@uhl-tr.nhs.uk

\section{References}

1 Corris P. Notice of duplicate publication. Clin Med 2001;1:430.

2 Editorial. Notice of duplicate publication. Thorax 2002;57:6

3 Warrell DA. To search and studdy out the secrett of tropical diseases by way of experiment. Clin Med 2001;1:485-94.

4 Warrell DA. To search and studdy out the secrett of tropical diseases by way of experiment. Lancet 2001;358:1983-8.
It is a matter of some concern to us that you felt obliged to print a notice of duplicate publication for Professor Corris. ${ }^{1}$ While we al deplore dual publication of original scientific data, the purpose of review articles is to provide a form of CME for practising physicians. It is therefore inevitable that, when an authority in a field is asked to give their current view on a subject, there will be considerable overlap with his/her previous thoughts on the subject. This does not make the article uninteresting to read, nor-as we are sure the Editors are aware-does it stop such articles being frequently referenced.

It is our belief that it is generally understood within the community that review articles by a given author are likely to contain significant overlap with previously published reviews by the same author and that, in this situation, it is rather "missing the point" to call this a duplicate publication.

To illustrate the point we enclose a list of review articles which all contain overlapping material concerning the assessment of respiratory muscle strength. ${ }^{2-8}$ With the exception of the article in Thorax (for which the invitation to write came following a prompt from us), the remaining articles were all written as a result of unsolicited requests by the editorial team of the journal concerned. Like Professor Corris's articles, they serve a useful function because these journals reach widely differing audiences and in each case the text of the article has been aligned to fit the interests of the readership of the journal concerned.

Our belief is that reviews of this sort do serve a useful role in postgraduate medical education and, because writing them is not recognised by the University Research Assessment Exercise, it is becoming increasingly hard to find experts in their fields who are prepared to do so. Publicly identifying this type of "duplicate publication" serves no useful purpose.

M Polkey

Consultant Physician, Royal Brompton Hospital London, UK

J Moxham

Professor of Respiratory Medicine, Guy's Kings and St Thomas' School of Medicine, London, UK

Correspondence to: $\operatorname{Dr} M$ Polkey, Respiratory Muscle Laboratory, Royal Brompton Hospital London SW3 6HP, UK m.polkey@rbh.nthames.nhs.uk

\section{References}

1 Anon. Notice of duplicate publication Thorax 2002;57:6

2 Polkey MI, Moxham J. Clinical aspects of respiratory muscle dysfunction in the critically ill. Chest 2001;119:926-39.

3 Polkey MI, Lyall RA, Moxham J, et al. Respiratory aspects of neurological disease. J Neurol Neurosurg Psychiatry 1999;66:5-15.

4 Polkey MI, Green M, Moxham J. Measurement of respiratory muscle strength. Thorax 1995:50:1 131-5.

5 Hart N, Polkey Ml. Investigation of respiratory muscle function. Clin Pulm Med 2001;8:180-7.

6 Polkey MI. Respiratory muscle disease: worth buying some equipment? Eur Respir Buyers 2000;3:9-11

7 Polkey MI. Respiratory muscle disease: when to suspect it and how to rule it out. Resp Dis Pract 2000;17:11-14.

8 Polkey MI, Moxham J. Terminology and testing of respiratory muscle dysfunction. Monaldi Arch Chest Dis 1999;54:514-9.

\section{Editors' reply}

We published the statement on duplicate publication, as did the other journal concerned, in response to a correspondent who pointed out the similarity between the two articles. When we looked through the article published in Clinical Medicine it was evident that large parts of the article in Thorax were reproduced verbatim.

We appreciate that review articles by the same author in different journals often contain overlapping information, but that was not the point on this occasion. The point was that much of the material was exactly the same, and Professor Corris had not explicitly acknowledged this or the contribution of other authors to it.

We have taken a firm line on duplicate publication and non-disclosure of related publications in the past and, although we accept that some degree of duplicate reporting is acceptable and common in review or opinion articles, having received a formal complaint about the article we did not feel able to dismiss it. This was particularly the case since Professor Corris was until very recently an Associate Editor of Thorax, and we were in danger of being open to accusations of special privilege for people who have been associated with the journal.

J Britton Executive Editor

\section{Chlamydia pneumoniae and COPD exacerbation}

We read with interest the recent paper by Blasi et al which showed that Chlamydia pneumoniae infection is associated with higher rates of exacerbation and airway microbial colonisation in patients with COPD. ${ }^{1}$ We have prospectively studied patients in the East London COPD study with daily monitoring using diary cards to detect COPD exacerbation defined using the same criteria. $^{23}$ Serum microimmunofluorescence (MIF) immunoglobulin $\mathrm{G}$ (IgG) titres for $C$ pneumoniae were measured in 110 patients ( $\mathrm{FEV} \% 41.7$ (18.4)) with stable COPD during 1 year with simultaneous estimation of plasma fibrinogen and serum interleukin 6 (IL-6); $26 \%$ of the patients had IgG titres of $\geqslant 1$ in 16 (fig 1). High $C$ pneumoniae IgG titres were not related to $\mathrm{FEV}_{1} \%$ predicted, exacerbation frequency, plasma fibrinogen, or serum IL-6 levels. In their paper Blasi et al did not report whether there was a relation between MIF titres and exacerbation frequency.

Blasi and colleagues found that $43 \%$ of patients when stable were positive for $C$ pneumoniae by DNA polymerase chain reaction (PCR) using peripheral blood mononuclear cells (PBMCs). At exacerbation they have only shown data for the 34 (of 61) who consented to the antibiotic trial and all 34 were positive for $C$ pneumoniae. In our study a further 33 patients FEV \% 39.8 (16.3)) were simultaneously sampled using nasopharyngeal aspirates and induced sputum when stable and during 43 COPD exacerbations. We found no $C$ pneumoniae using a nested reverse transcriptase PCR adapted from Cunningham et $a l^{4}$ at stable baseline but nine patients (seven from induced sputum and another two in nasal aspirates) 


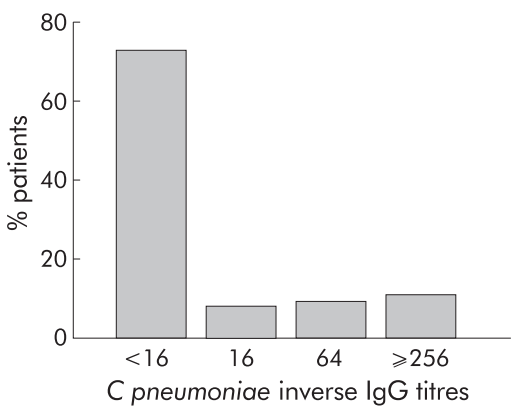

Figure 1 Distribution of serum $C$ pneumoniae microimmunofluorescence (MIF) IgG antibody inverse titres in 110 patients with stable COPD.

$(28 \%)$ were positive for $C$ pneumoniae at exacerbation. The presence of $C$ pneumoniae was not associated with smoking history, $\mathrm{FEV}_{1} \%$, peak flow change at or peak flow recovery from COPD exacerbation, rate of peak flow recovery, IL-6 (fig 2) or IL-8 levels, or total and differential cell counts in induced sputum. The lack of relationship between $C$ pneumoniae detection and inflammatory markers at exacerbation suggests to us that $C$ pneumoniae exacerbations are no different from exacerbations not associated with $C$ pneumoniae.

We found no relationship between $C$ pneumoniae detection in the airway at exacerbation and exacerbation frequency $(p=0.504)$, but Blasi et al found that $C$ pneumoniae positive patients (in stable COPD) had a greate tendency towards frequent exacerbation. However, the difference in exacerbation frequency between the two groups was small ( 0.6 exacerbations per year), and the authors need to be cautious about concluding that this difference could affect disease progression.

The main difference between the data of Blasi et al and ours is that in their study 16 of 42 patients $(38 \%)$ enrolled in study 1 had sputum positive for $C$ pneumoniae by DNA PCR and a similar number $(61 / 141,43 \%)$ in study 2 in PBMCs, both during stable COPD. We sampled only once in stable COPD and found none, despite finding $28 \%$ at exacerbation. Blasi and colleagues sampled subjects repeatedly (at least four times), but it is not clear how many times they had to be positive to be defined as "respiratory samples positive for $C$ pneumoniae by DNA PCR". The 16 positive patients provided 69 sputum samples; were all sputum samples positive on all occasions examined in these patients? Similarly, were al 125 sputum samples from the 26 patients who

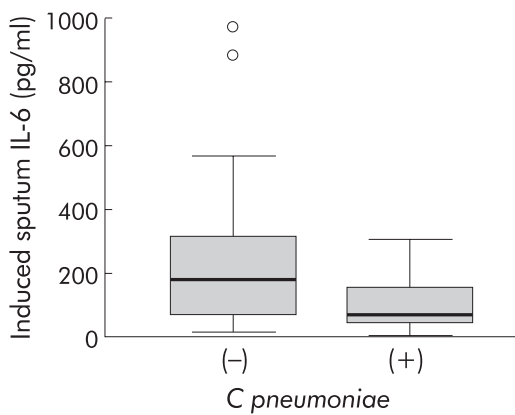

Figure 2 Induced sputum levels of IL-6 during Chlamydia (+) and non-Chlamydia (-) COPD exacerbations in 33 patients with 43 exacerbations. Outliers are shown; $p=0.187$ (Mann-Whitney U test). were $C$ pneumoniae negative always negative? It would be helpful if the authors could give the data on the chronic nature of infection in their sputum samples.

T A R Seemungal, J A Wedzicha Academic Unit of Respiratory Medicine, St Bartholomew's and Royal London School of Medicine and Dentistry, London

P K MacCallum

Medical Research Council Epidemiology and Medical Care Unit, Wolfson Institute of Preventive Medicine, St Bartholomew's and Royal London School of Medicine and Dentistry, London

S L Johnston

Imperial College at St Mary's Hospital, London

P A Lambert

Aston University, Birmingham

Correspondence to: Professor J A Wedzicha, Academic Unit of Respiratory Medicine, Dominion House, St Bartholomew's Hospital, London EClA 7BE, UK; J.A.Wedzicha@qmul.ac.uk

\section{References}

1 Blasi F, Damato S, Cosentini R, et al. Chlamydia pneumoniae and chronic bronchitis: association with severity and bacterial clearance following treatment. Thorax 2002;57:672-6.

2 Seemungal TAR, Donaldson GC, Paul EA, et al. Effect of exacerbation on quality of life in patients with chronic obstructive pulmonary disease. Am J Respir Crit Care Med

1998;157:1418-22.

3 Anthonisen NR, Manfreda J, Warren CP. Antibiotic therapy in exacerbations of chronic obstructive pulmonary disease. Ann Intern Med 1987:106:196-204.

4 Cunningham AF, Johnston SL, Julious SA. Chronic Chlamydia pneumoniae infection and asthma exacerbations in children. Eur Respir J 1998;11:345-9.

\section{Authors' reply}

We are grateful to Seemungal et al for their comments regarding our recently published paper on Chlamydia pneumoniae and chronic bronchitis.

Seemungal et al prospectively studied 110 patients with COPD for 1 year, evaluating serum microimmunofluorescence IgG titres, plasma fibrinogen, and IL-6 levels. They found no correlation between high IgG titres and FEV $\%$ predicted exacerbation frequency, plasma fibrinogen, and serum IL-6 levels. We also found no correlation between serological results and $\mathrm{FEV}_{1} \%$ predicted or exacerbation frequency. In fact, as in previous reports, ${ }^{2}$ we found a low degree of correlation between $C$ pneumoniae serology and peripheral blood mononuclear cell (PBMC) PCR. A greater degree of correlation was observed when IgG and IgA titres were combined but, unfortunately, no comparison is possible as Seemungal et al only performed IgG titre determinations. In any case, our findings are not truly comparable with those of Seemungal et al as serology is known to be less specific than PCR for the identification of chronic infection with $C$ pneumoniae. ${ }^{3}$

In the second part of their letter Seemungal et al report the results of an analysis on a further group of 33 patients who were simultaneously sampled for nasal aspirates and induced sputum when stable and during exacerbation. They found no PCR positivity in stable patients, whereas in nine of 43 exacerbations C pneumoniae was detected by PCR in respiratory specimens. The authors infer that DNA positivity in the sputum is a marker of $C$ pneumoniae acute infection; this would mean that around $30 \%$ of all acute exacerbations are sustained by $C$ pneumoniae. However, the gold standard for acute infection is still considered serology on paired samples. Applying both PCR and serology on paired serum samples we found an acute infection in two of 34 exacerbations confirming our previous data of an overall incidence of 5-6\%. ${ }^{4}$ Their definition of acute $C$ pneumoniae infection may explain, at least in part, why they could not detect any difference between exacerbations associated or not associated with $C$ pneumoniae in terms of inflammatory response.

The reported discrepancy in PCR positivity on respiratory samples between our study and that of Seemungal et al may be related to different PCR techniques. In fact, we found 16/42 (38\%) PCR positive patients with stable COPD, whereas they found $0 / 33$ and $9 / 33$ $(28 \%)$ in stable COPD and during an exacerbation, respectively. Considering that the rate of positivity in our stable patients is comparable to that of patients with exacerbation in Seemungal's series, we think that the different PCR results may simply be related to PCR sensitivity, sputum quality/quantity, amount of DNA retrieved from the samples, and number of tested samples. ${ }^{5}$ Seemungal et al tested a single induced sputum specimen for each stable patient whereas we analysed at least four spontaneous sputum samples for each stable patient. We defined any patient with at least two positive specimens as PCR positive. Sixteen patients were classified as PCR positive on the basis of $43 / 69$ (62\%) PCR positive sputum samples. Twenty six patients were considered PCR negative; 24 had repeatedly negative PCR results on all 113 sputum samples and two patients had a single PCR positive sputum specimen (2/12 specimens).

Our results on exacerbation frequency are based on the observation of 141 subjects for 2 years compared with 33 subjects in the study by Seemungal $e t$ al. The different number of subjects included in the two studies may explain some discrepancies. We do agree that caution is needed in interpreting the results of our study and stated that "our study indicates the possible role of $C$ pneumoniae chronic infection in disease progression in COPD patients. Further confirmation based on large scale trials is needed". ${ }^{1}$ However, even a slight increase in exacerbation frequency may have a role in disease progression. ${ }^{67}$

F Blasi, L Allegra

Institute of Respiratory Diseases, University of Milan, IRCCS Ospedale Maggiore Milano, Italy

S Damato, R Raccanelli Division of Pneumology, University of Milan Bicocca, Ospedale di Seregno, Italy

R Cosentini, P Tarsia

Department of Emergency Medicine, IRCCS Ospedale Maggiore Milano, Italy

S Centanni

Respiratory Unit, Institute of Lung Disease Ospedale San Paolo, University of Milan, Italy

Correspondence to: Dr F Blasi, Istituto di Tisiologia e Malattie dell'Apparato Respiratorio, Università degli Studi di Milano, IRCCS Ospedale Maggiore di Milano, I-20122 Milano, Italy; francesco.blasi@unimi.it

\section{References}

1 Blasi F, Damato S, Cosentini R, et al Chlamydia pneumoniae and chronic bronchitis: association with severity and bacterial clearance following treatment. Thorax 2002; 57:672-6.

2 Blasi F, Boman J, Esposito G, et al. Chlamydia pneumoniae DNA detection in peripheral blood mononuclear cells is predictive of vascular infection. J Infect Dis 1999; 180:2074-6. 
3 Dowell SF, Peeling RW, Boman J, et al. Standardizing Chlamydia pneumoniae assays: recommendations from the Centers for Disease Control and Prevention USA and Laboratory Center for Disease Control Canada. Clin Infect Dis 2001;33:492-502.

4 Blasi F, Legnani D, Lombardo V, et al. Chlamydia pneumoniae infection in acute exacerbations of COPD. Eur Respir J 1993;6:19-22

5 Apfalter P, Blasi F, Boman J, et al. Multicenter comparison trial of DNA extraction methods and PCR assays for detection of Chlamydia pneumoniae in endarterectomy specimens. J Clin Microbiol 2001;39:519-24.

6 Kanner RE, Anthonisen NR, Connett JE, et al. Lower respiratory illnesses promote FEV 1 decline in current smokers but not ex-smokers with mild chronic obstructive pulmonary disease. Am J Respir Crit Care Med 2001;164:358-64.

7 Donaldson GC, Seemungal TAR, Bhowmik $A$, et al. Relationship between exacerbation frequency and lung function decline in chronic obstructive pulmonary disease. Thorax 2002;57:847-52.

\section{ICU outcomes in acute respiratory failure secondary to COPD}

The data presented by Breen et al ${ }^{1}$ regarding the outcomes of patients with chronic obstructive pulmonary disease (COPD) are encouraging and lend support to the respiratory physician often faced with nihilistic attitudes towards ventilating these patients in acute respiratory failure. However, despite the proposition by the authors that certain patients with likely poor outcomes might have been excluded, the ICU stays for both groups (intubated and non-intubated) are strikingly short.

This suggests that the threshold for intubation as opposed to non-invasive ventilation (NIV) may have been lower before 1994 than in current practice. Although the authors explain the reason for the high levels of $\mathrm{Pao}_{2}$ on admission to the ICU, it could be that the severity of respiratory acidosis may have reflected excess oxygen therapy rather than the severity of the underlying mechanical respiratory failure, thus being more readily reversible and requiring a shorter period of ventilatory support. Although the decision to intubate is not solely based upon blood gases, with the increasing availability of NIV it might be that a subgroup of these patients would now be managed using controlled oxygen therapy, respiratory stimulants, and NIV.

As a result, I suspect that the physiological state of the patient that we offer to the ICU in our current practice may be worse than in this study with commensurate outcomes (longer stays and higher mortality). Despite this, many patients still do well and studies of this type need to continue to assess predictors of unfavourable end points.

L Howard

Department of Respiratory Medicine, Norfolk \& Norwich University Hospital, Norwich NR4 7UY UK; luke.howard@tinyworld.co.uk

\section{References}

1 Breen D, Churches T, Hawker F, et al. Acute respiratory failure secondary to chronic obstructive pulmonary disease treated in the intensive care unit: a long term follow up study. Thorax 2002;57:29-33.

2 Plant PK, Owen JL, Elliott MW. Early use of non-invasive ventilation for acute exacerbations of chronic obstructive pulmonary disease on general respiratory wards: a multicentre randomised controlled trial. Lancet 2000;355:1931-5.

\section{Marginal benefits of adding formoterol}

Price and colleagues ${ }^{1}$ conclude that adding formoterol confers a therapeutic advantage to inhaled steroid in patients with mild to moderate asthma. During the 6 month follow up, in part II of the study the frequency of the secondary outcome of mild asthma exacerbations differed by 2.5 per patient per 6 months while the difference in poorly controlled asthma days was 4.2 days per patient per 6 months. These differences, while statistically significant, are unlikely to be of real clinical relevance. Indeed, during the same period the difference in quality of life was neither significant nor clinically relevant. The main differences which were significant were in bronchodilator sensitive outcomes such as peak flow and reliever use, which are to be expected when patients are taking a 24/7 bronchodilator. These data are little differen from those in steroid naive patients in the OPTIMA trial over 12 months where the addition of formoterol to low dose budesonide improved lung function but not exacerbations, while in the same trial the addition of formoterol conferred only a small but significant reduction in exacerbations in patients previously treated with corticosteroids. ${ }^{2}$

Pointedly, neither of these studies evaluated any inflammatory surrogates. We would therefore suggest that these trials indicate that most patients with mild to moderate asthma can be adequately controlled on low to medium doses of inhaled budesonide alone, and that there is only a marginal advantage conferred by adding formoterol. Moreover, combination inhalers are considerably more expensive than inhaled steroid alone and their routine use is not warranted in primary care.

B J Lipworth, C M Jackson

Asthma and Allergy Research Group, Ninewells University Hospital, Dundee, UK Correspondence to: Professor B J Lipworth, Asthma and Allergy Research Group, Ninewells University Hospital, Dundee DD1 9SY, UK; topdoc@brianlipworth.com

\section{Reference}

1 Price D, Dutchman D, Mawson AA, et al on behalf of the FLOW (Eformoterol in the management of mild asthma - eformoterol Turbohaler with budesonide Turbohaler) Research Group. Early asthma control and maintenance with eformoterol following reduction of inhaled corticosteroid dose. Thorax 2002;57:791-8.

2 O'Byrne PM, Barnes PJ, Rodriguez-Roisin R, et al. Low dose inhaled budesonide and formoterol in mild persistent asthma: the OPTIMA randomized trial. Am J Respir Crit Care Med 2001;164:1392-7.

\section{BOOK REVIEW}

\section{Respiratory Medicine Specialist Handbook}

P Dilworth, D R Baldwin. UK: Harwood Academic Publishers, 2001. £39.50. ISBN 9058230775

This is the first in a new series of specialist handbooks that aims to fill the niche between the comprehensive textbook and the pocke handbook. There are the obvious pitfalls of trying to squeeze in too much detail at the expense of accessibility or reducing the subject to little more than a series of disjointed notes. However, this book-for the most part-steers clear of both of these errors and has produced a very readable, yet reasonably detailed, summary of specialist respiratory medicine. The 31 chapters cover a wide variety of topics and the authors' list is like a "Who's Who?" of UK respiratory medicine.

It is possible to pick up this book, read a chapter in less than half an hour, and come away with an increased knowledge of the pathophysiology of the condition under study and, perhaps more usefully, the intricacies of practical management which is the focus of the book. It will therefore cater to the specialist registrar undergoing higher specialist training in providing a broad understanding in reasonable detail of most facets of respiratory medicine, but it could also be of use to the experienced physician in reaffirming, reminding, and refreshing of the basics, and perhaps updating knowledge with regard to more recent developments.

The book is attractively presented with short paragraphs of text interspersed with helpful tables and figures. For those who are stimulated to seek more information on any subject, each chapter has a selection of references for further reading. Whilst we all might aspire to read, study and inwardly digest a weighty, comprehensive tome of respiratory medicine, for most of us in busy clinical practice this proves difficult. This reviewer would therefore encourage reading and studying this excellent book as an alternative which is more attainable and possibly of more practical relevance.

T J Warke

Department of Respiratory Medicine, Belfast City Hospital, Belfast BT9 7AB, UK; tim@dsl.pipex.com

\section{NOTICE}

\section{Scadding-Morriston Davies Joint Fellowship in Respiratory Medicine 2003}

This fellowship is available to support visits to medical centres in the UK or abroad for the purpose of undertaking studies related to respiratory medicine. Applications are invited from medical graduates practising in the UK, including consultants and irrespective of the number of years in that grade. There is no application form but a curriculum vitae should be submitted together with a detailed account of the duration and nature of the work and the centres to be visited, confirming that these have agreed to provide the facilities required. Please state the sum of money needed for travel and subsistence. A sum of up to $£ 20000$ can be awarded to the successful candidate, or the sum may be divided to support two or more applications. Applications should be sent to Dr I A Campbell, Secretary to the Scadding-Morriston Davies Fellowship, Llandough Hospital, Penarth, Vale of Glamorgan CF64 2XX, UK by 31 January 2003. 